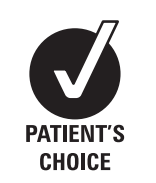

${ }^{1}$ Department of Neurology, University Medical Center Groningen, University of Groningen, Groningen, The Netherlands; ${ }^{2}$ Department of Neurology, UZ Brussel, Vrije Universiteit Brussel, Brussels, Belgium

Correspondence to:

Dr M Koch, Department of

Neurology, University Medical Center Groningen, Postbus 30.

001, 9700RB Groningen, The Netherlands;

m.w.koch@neuro.umcg.nl

Received 15 August 2008 Revised 6 October 2008

Accepted 3 November 2008

\title{
Parity and secondary progression in multiple sclerosis
}

\author{
M Koch, ${ }^{1}$ M Uyttenboogaart, ${ }^{1}$ D Heersema, ${ }^{1}$ C Steen, ${ }^{1}$ J De Keyser ${ }^{1,2}$
}

\begin{abstract}
Background: Pregnancy has a well-documented effect on relapses in multiple sclerosis (MS), whereas little is known about the impact of pregnancy and childbirth on the risk of secondary progression.

Objective: To investigate the association of parity and secondary progression in women with MS.

Methods: The association of the number of births and secondary progression was studied in a hospital-based cohort of 277 women with MS. Data were analysed in a multivariable logistic regression model, with adjustment for possible confounders.

Results: Parity was not independently associated with secondary progression, while the factors disease duration (OR per year increase: $1.05,95 \% \mathrm{Cl} 1.03$ to 1.09 ) and use of immunomodulatory treatments (OR $0.23,95 \% \mathrm{Cl} 0.08$ to 0.65 ) were independently associated with secondary progression.

Conclusion: We found no evidence that parity influences the risk of secondary progression in MS. Further population-based studies on the association of pregnancy and childbirth on the long-term prognosis of MS are needed.
\end{abstract}

Multiple sclerosis (MS) affects women about twice as often as men. Since MS often begins in early adulthood, the course of the disease overlaps the childbearing years in many women with MS. A number of studies have been concerned with the impact of pregnancy on MS relapses, but little research has been done on the influence of pregnancies on the onset of secondary progression.

The pathophysiological mechanisms underlying relapses are focal inflammatory lesions in the central nervous system (CNS). The main pathophysiological mechanism of secondary progression appears to be an ill-understood diffuse neurodegeneration throughout the CNS termed "axonal degeneration." Axonal degeneration may begin early in the disease but becomes apparent as secondary progression when enough axons have degenerated to exhaust the functional reserve capacity of the CNS. ${ }^{12}$

Previous studies have shown that relapses occur less frequently during pregnancy, especially during the third trimester, and that there is a rebound effect with an increase in relapse frequency after delivery. ${ }^{3}$ These findings have been associated with the rise and fall of oestrogen and progesterone levels over the course of pregnancy and argue for a suppressive effect of these steroid hormones on the inflammatory component of MS. One exploratory crossover trial found evidence for an effect of oral oestriol treatment on the number of enhancing MRI lesions in a group of 12 women with MS. ${ }^{4}$
Information about the effect of pregnancy on the development of secondary progression is scarce. We found only one small study suggesting a significantly decreased risk of a progressive course in women who became pregnant after MS onset. ${ }^{5}$

We performed a multivariable logistic regression analysis to evaluate whether having given birth (parity) independently affects the risk of secondary progression in MS.

\section{PATIENTS AND METHODS \\ Patients}

The data used in this study were taken from the Groningen MS database. This database contains data on all MS patients attending the MS clinic at the University Medical Center Groningen. At this clinic, patients are seen at least once a year, and demographic and disease-specific data are entered into an electronic database. All patients have a diagnosis of definite MS according to the Poser criteria. ${ }^{6}$ Secondary progression was defined as continuous worsening of neurological symptoms unrelated to relapse for at least 1 year. ${ }^{7}$ The data include the time of disease onset, symptom at disease onset, use of immunomodulatory treatment (IMT), the onset of secondary progression and the number and birth dates of children.

\section{Statistical considerations}

The most attractive method to analyse time to event data, such as the time to secondary progression, is by using multivariable Cox regression models. However, Cox regression analysis requires that the so-called "proportional hazards assumption" holds, which in our case means that pregnancies ending in childbirth are expected to have the same effect on the risk of developing secondary progression over the entire course of follow-up. Since pregnancy is restricted to the fertile period of a patient, the proportional hazards assumption is violated, and there is no easy way to correct for this. Because of this difficulty, we decided to perform a binary logistic regression analysis, which is a relatively straightforward method to estimate the independent association of parity and the development of secondary progression with correction for possible confounders, such as age at disease onset, disease duration, symptom at disease onset and the use of IMT.

\section{Regression models}

The data were entered into a multivariable binary logistic regression model with secondary progression as the dependent variable. Parity after disease onset ("parous" vs "nulliparous"), age at disease onset, disease duration, the use of IMT (for at least 
Table 1 Multivariable logistic regression model of factors associated with secondary progression

\begin{tabular}{llc}
\hline Factor & Odds ratio (95\% CI) & p Value \\
\hline $\begin{array}{ll}\text { Parity } \\
\quad \text { Nulliparous }\end{array}$ & (Reference) & 0.81 \\
$\quad$ Parous & $0.93(0.50$ to 1.72$)$ & 0.27 \\
Age at disease onset* & $0.98(0.95$ to 1.01$)$ & $<0.0005$ \\
Disease duration* & $1.05(1.03$ to 1.09$)$ & \\
Immunomodulatory & & 0.005 \\
treatment use $\dagger$ & & \\
No & (Reference) & 0.52 \\
$\quad$ Yes & $0.23(0.08$ to 0.65$)$ & \\
Symptom at onset & & \\
Motor & (Reference) & \\
Sensory & $1.00(0.43$ to 2.37$)$ & \\
Brainstem/cerebellar & $1.34(0.46$ to 3.84$)$ & \\
Visual & $1.67(0.71$ to 3.94$)$ & \\
Other & $1.05(0.24$ to 4.67$)$ &
\end{tabular}

Hosmer and Lemeshow $p=0.13$.

*Per year increase.

$\dagger$ For at least 1 year before secondary progression or censoring.

1 year before development of secondary progression or inclusion) and the symptom at disease onset were entered as covariates.

The possible effect of multiparity was investigated in a further model in which parity was classified as "nulliparous" versus "one child" versus "two or more children." The effect of the total number of pregnancies (before and after the onset of MS) was investigated in a similar model.

The goodness of fit of all regression models was assessed with the Hosmer and Lemeshow statistic.

Statistical significance was taken to be at the two-tailed 0.05 level. All statistical analyses were performed with the SPSS statistical software package version 14 (SPSS, Chicago).

\section{RESULTS}

\section{Study population}

Information on parity was available in 277 patients with a relapsing-remitting disease onset. The median age at disease onset was 27 years (interquartile range, IOR 23 to 34), and the median disease duration was 17 years (IOR 12 to 25). A total of 136 patients (49\%) had developed secondary progression.

For each patient, we determined the number of children born after the onset of $\mathrm{MS}$ and before the onset of secondary progression or inclusion into this study: 197 (71\%) bore no children during this period, $34(12 \%)$ had one child, and 46 (17\%) gave birth to two or more children.

Further information used in this study was the use of IMT for at least 1 year before the onset of secondary progression or before inclusion in this study (present in 29 patients or $11 \%$ of the population), and the symptom at disease onset (categorised as "motor" in 32 (12\%), "sensory" in 103 (37\%), "brainstem/ cerebellar" in 31 (11\%), "visual" in 99 (36\%) and as "other" in 12 (4\%) patients).

\section{Regression models}

Parity was not independently associated with secondary progression in our patient cohort (odds ratio $(\mathrm{OR})$ of parous versus nulliparous women: $0.93,95 \%$ CI 0.50 to 1.72$)$. The use of IMT decreased the odds (OR $0.23,95 \%$ CI 0.08 to 0.65 ) and disease duration increased the odds (OR per year increase 1.05,
95\% CI 1.03 to 1.09) of secondary progression (table 1). Repeating these analyses with the number of children categorised as "nulliparous," "one child" and "two or more children" delivered comparable results. Repeating the analyses with the total number of children (born before or after the onset of MS) also gave comparable results (data not shown).

The Hosmer and Lemeshow statistic indicated that all regression models had acceptable goodness of fit.

\section{DISCUSSION}

In our patient cohort, parity was not independently associated with secondary progression. Thus, patients who had given birth were neither more nor less likely to develop secondary progression.

The literature on the effect of pregnancy and childbirth on long-term outcomes in MS is dominated by studies investigating disability (for review see Damek and Shuster) ${ }^{8}$ and controversial. Some of these studies found no effect of pregnancy on the accumulation of disability, ${ }^{9-11}$ while others even suggested a beneficial effect. ${ }^{5}{ }^{12}$ The only other study that assessed the development of secondary progression compared 28 parous patients with 55 nulliparous patients from a Swedish MS cohort. The authors defined 16 groups, matched for disease duration and level of disability, and found a later onset of secondary progression in the parous patients $(p=0.024) .{ }^{5}$ These findings are in conflict with ours, but the Swedish study had a smaller number of patients and used a less stringent statistical approach. However, both studies agree that there is no negative influence of pregnancy and childbirth on the risk of conversion from a relapsing-remitting to a chronic progressive disease course.

We and others have recently found some indication that the use of IMT may postpone the development of secondary progression. ${ }^{13}{ }^{14}$ This effect may be due to the anti-inflammatory action of IMT, which could preserve part of the functional reserve capacity of the CNS. However, patients who stop their medication because of an aggressive disease course in spite of IMT use may have biased these findings. The immunosuppressive state of pregnancies is not reflected in a lower risk of secondary progression in our study, possibly because the effect of pregnancy is neutralised by a rebound effect during the puerperium.

Potential limitations of our study are the fact that our database is hospital-based rather than population-based, which could limit the applicability of our findings to the whole population of MS patients. Besides, we only recorded full-term pregnancies leading to a live birth, so that we could not investigate the effect of pregnancies ending in abortion, miscarriage or stillbirth. Another limitation is the influence of the disease course on the choice of a patient to bear children. Women who develop secondary progression may regularly choose to have no or fewer children-and this would associate progression with nulliparity or few pregnancies. This association could, if strong enough, render a genuine beneficial effect of parity on secondary progression undetectable.

Further studies on the effect of pregnancy on the longer-term prognosis of MS are warranted. Such research should preferably be performed in large population-based cohorts.

Funding: MK is an "MD-clinical research trainee" supported by The Netherlands Organization for Health Research and Development (The Hague, The Netherlands) and MS Anders (Amsterdam, The Netherlands).

Competing interests: None. 


\section{REFERENCES}

1. Bjartmar C, Wujek JR, Trapp BD. Axonal loss in the pathology of MS: consequences for understanding the progressive phase of the disease. J Neurol Sci 2003;206:165-71.

2. Lassmann H. Axonal injury in multiple sclerosis. J Neurol Neurosurg Psychiatry 2003;74:695-7.

3. Confavreux C, Hutchinson M, Hours MM, et al. Rate of pregnancy-related relapse in multiple sclerosis. Pregnancy in Multiple Sclerosis Group. N Engl J Med 1998;339:285-91.

4. Sicotte NL, Liva SM, Klutch $\mathrm{R}$, et al. Treatment of multiple sclerosis with the pregnancy hormone estriol. Ann Neurol 2002;52:421-8.

5. Runmarker B, Andersen 0. Pregnancy is associated with a lower risk of onset and a better prognosis in multiple sclerosis. Brain 1995:118:253-61.

6. Poser CM, Paty DW, Scheinberg L, et al. New diagnostic criteria for multiple sclerosis: guidelines for research protocols. Ann Neurol 1983;13:227-31.
7. Kremenchutzky M, Rice GPA, Baskerville J, et al. The natural history of multiple sclerosis: a geographically based study 9: observations on the progressive phase of the disease. Brain 2006;129:584-94.

8. Damek DM, Shuster EA. Pregnancy and multiple sclerosis. Mayo Clin Proc 1997;72:977-89.

9. Poser S, Poser W. Multiple sclerosis and gestation. Neurology 1983;33:1422-7

10. Thompson DS, Nelson LM, Burns A, et al. The effects of pregnancy in multiple sclerosis: a retrospective study. Neurology 1986;36:1097-9.

11. Weinshenker BG, Hader W, Carriere W, et al. The influence of pregnancy on disability from multiple sclerosis: a population-based study in Middlesex County, Ontario. Neurology 1989;39:1438-40.

12. Verdru P, Theys $P$, D'Hooghe $M B$, et al. Pregnancy and multiple sclerosis: the influence on long term disability. Clin Neurol Neurosurg 1994;96:38-41.

13. Trojano M, Pellegrini F, Fuiani A, et al. New natural history of interferon-beta-treated relapsing multiple sclerosis. Ann Neurol 2007:61:300-6.

14. Koch M, Uyttenboogaart M, van HA, et al. Factors associated with the risk of secondary progression in multiple sclerosis. Mult Scler 2008;14:799-803.

\section{Quality \& Safety in Health Care}

Quality \& Safety in Health Care is a leading international peer-review journal in the growing area of quality and safety improvement. It provides essential information for those wanting to reduce harm and improve patient safety and the quality of care. The journal reports and reflects research, improvement initiatives and viewpoints and other discursive papers relevant to these crucial aims with contributions from researchers, clinical professionals and managers and experts in organisational development and behaviour.

qshc.bmj.com

Quality \&Safety in 